Facial Asymmetry in Nonsyndromic and Muenke

Syndrome-Associated Unicoronal Synostosis : A 3-Dimensional

Study Based on Facial Surfaces Extracted From CT Scans

Owall, Louise

2021-06

Owall , L , Darvann , T A , Hove , H B , Heliövaara , A , Duno , M , Kreiborg , S \& Hermann , N 2021, ' Facial Asymmetry in Nonsyndromic and Muenke Syndrome-Associated Unicoronal Synostosis : A 3-Dimensional Study Based on Facial Surfaces Extracted From CT Scans ' , Cleft Palate - Craniofacial Journal , vol. 58 , no. 6 , pp. 687-696 . https://doi.org/10.1177/105566562095

http://hdl.handle.net/10138/332979

https://doi.org/10.1177/1055665620959983

cc_by_nc

publishedVersion

Downloaded from Helda, University of Helsinki institutional repository.

This is an electronic reprint of the original article.

This reprint may differ from the original in pagination and typographic detail.

Please cite the original version. 


\title{
Facial Asymmetry in Nonsyndromic and Muenke Syndrome-Associated Unicoronal Synostosis: A 3-Dimensional Study Based on Facial Surfaces Extracted From CT Scans
}

The Cleft Palate-Craniofacial Journal 202I, Vol. 58(6) 687-696 (c) 2020, American Cleft PalateCraniofacial Association Article reuse guidelines: sagepub.com/journals-permissions DOI: I0.1 I 177// 055665620959983 journals.sagepub.com/home/cpc @SAGE

\author{
Louise Öwall, MD ${ }^{1}$, Tron A. Darvann, MSc, PhD ${ }^{1,2}$, Hanne B. Hove, MD, DMSc ${ }^{3,4}$, \\ Arja Heliövaara, DDS, PhD $^{5}$, Morten Dunø, ErCLG, PhD 6 , \\ Sven Kreiborg, DDS, PhD, Dr Odont ${ }^{1,7}$, and Nuno V. Hermann, DDS, PhD, Dr Odont ${ }^{1,7}$
}

\begin{abstract}
Objective: To quantify soft tissue facial asymmetry (FA) in children with nonsyndromic and Muenke syndrome-associated unicoronal synostosis (NS-UCS and MS-UCS), hypothesizing that MS-UCS presents with significantly larger FA than NS-UCS.

Design: Retrospective cohort study.

Patients and Methods: Twenty-one children (mean age: 0.6 years; range: $0.1-1.4$ years) were included in the study (NS-UCS = I4; MS-UCS = 7). From presurgical computed tomography scans, facial surfaces were constructed for analysis. A landmark guided atlas was deformed to match each patient's surface, obtaining spatially detailed left-right point correspondence. Facial asymmetry was calculated in each surface point across the face, as the length $(\mathrm{mm})$ of an asymmetry vector, with its Cartesian components providing 3 directions. Mean FA was calculated for the full face, and the forehead, eye, nose, cheek, mouth, and chin regions.

Results: For the full face, a significant difference of $2.4 \mathrm{~mm}(P=.00 \mathrm{I})$ was calculated between the 2 groups, predominately in the transverse direction $(1.5 \mathrm{~mm} ; P<.00 \mathrm{I})$. The forehead and chin regions presented with the largest significant difference, $3.5 \mathrm{~mm}$ $(P=.002)$ and $3.2 \mathrm{~mm}(P<.00 \mathrm{I})$, respectively; followed by the eye $(2.4 \mathrm{~mm} ; P=.004)$, cheek $(2.2 \mathrm{~mm} ; P=.004)$, nose $(\mathrm{I} .7 \mathrm{~mm}$; $P=.00 \mathrm{I})$, and mouth $(\mathrm{I} .4 \mathrm{~mm} ; P=.009)$ regions. The transverse direction presented with the largest significant difference in the forehead, chin, mouth, and nose regions, the sagittal direction in the cheek region, and the vertical direction in the eye region. Conclusions: Muenke syndrome-associated unicoronal synostosis presented with significantly larger FA in all regions compared to NS-UCS. The largest significant differences were found in the forehead and chin regions, predominantly in the transverse direction.
\end{abstract}

\section{Keywords}

facial morphology, synostosis, pediatrics

\footnotetext{
I 3D Craniofacial Image Research Laboratory (School of Dentistry, University of Copenhagen, Center of Head and Orthopedics, Copenhagen University Hospital Rigshospitalet, and DTU Compute, Technical University of Denmark), Copenhagen, Denmark

${ }^{2}$ Department of Oral and Maxillofacial Surgery, Copenhagen University Hospital Rigshospitalet, Copenhagen, Denmark

${ }^{3}$ Center for Rare Diseases, Department of Pediatrics, Copenhagen University Hospital Rigshospitalet, Copenhagen, Denmark

${ }^{4}$ The RAREDIS Database, Center for Rare Diseases, Department of Pediatrics, Copenhagen University Hospital Rigshospitalet, Copenhagen, Denmark

${ }^{5}$ Cleft Palate and Craniofacial Center, Department of Plastic Surgery, Helsinki University Hospital, Helsinki, Finland

${ }^{6}$ Center for Rare Diseases, Department of Clinical Genetics, Copenhagen University Hospital Rigshospitalet, Copenhagen, Denmark

${ }^{7}$ Department of Pediatric Dentistry and Clinical Genetics, School of Dentistry, Faculty of Health and Medical Science, University of Copenhagen, Copenhagen, Denmark
} 


\section{Introduction}

Unicoronal synostosis (UCS) occurs as either an isolated sporadic malformation, where the underlying cause is most often unknown, or as part of a syndrome, for example, Muenke syndrome (MS). Muenke syndrome is caused by the p.Pro250Arg mutation in the FGFR3 gene and is transmitted in an autosomal dominant manner; however, high phenotypic variability characterizes this syndrome due to reduced penetrance and variable expressivity (Muenke et al., 1997; Passos-Bueno et al., 2008). The phenotype in MS ranges from asymptomatic cases and cases with isolated coronal synostosis (bilaterally or unilaterally) to multisymptomatic cases (Muenke et al., 1997; Kruszka et al., 2016; Öwall et al., 2020).

Unicoronal synostosis is a complex condition affecting the entire craniofacial region, from the primary anomaly of the neurocranium to the cranial base and the mandible (Kreiborg and Björk, 1981; Kreiborg et al., 1985; Marsh et al., 1986; Richtsmeier et al., 1991; Kane et al., 1996; Lo et al., 1996). Children born with UCS present with a face characterized by asymmetry in all 3 anatomical planes: transverse, vertical, and sagittal. Typical facial characteristics involve; a flat and retruded forehead on the ipsilateral side of the synostosis and frontal bossing on the contralateral side, an elevated (harlequin) eyebrow and a wider palpebral fissure on the ipsilateral side and a depressed eyebrow and narrower palpebral fissure on the contralateral side, deviation of the nasal root to the ipsilateral side and of the nasal tip and chin to the contralateral side (Cohen, 2000).

According to the golden standard guidelines, children with UCS should be treated surgically, preferably during the first year of life. The main goals of the surgical procedure are to prevent elevated intracranial pressure by expanding the intracranial volume and to reshape the cranial vault to improve the aesthetic appearance (Derderian and Seaward, 2012; Warren et al., 2012; Moderie et al., 2019). Regarding the surgical outcome, in terms of restoring a symmetrical facial appearance, several previous studies have found a poorer outcome and a higher reoperation rate in children with MS-associated UCS (MS-UCS) compared to nonsyndromic UCS (NS-UCS; Cassileth et al., 2001; Thomas et al., 2005; Honnebier et al., 2008; Öwall et al., 2016; Öwall et al., 2019). Furthermore, it has been documented that the midsagittal plane (MSP) is more deviated in MS-UCS, compared to NS-UCS (Keller et al., 2007).

The aim of this study was to compare the degree of soft tissue facial asymmetry (FA) in infants with NS-UCS and MS-UCS using a method capable of objective and detailed evaluation of FA in 3 dimensions (transverse, vertical, and sagittal). It was hypothesized that infants with MS-UCS present with a more severe degree of soft tissue FA compared to infants with NS-UCS.

\section{Materials and Methods}

\section{Study Sample}

Unoperated infants with UCS were allocated from craniofacial centers in Copenhagen and Aarhus, Denmark $(\mathrm{N}=15)$ and the
Helsinki Cleft Palate and Craniofacial Center, Helsinki, Finland $(\mathrm{N}=6)$. The total sample consisted of 21 infants with UCS (13 girls, 8 boys; mean age: 0.6 years; range: 0.1-1.4 years). All children included had UCS verified by computed tomography (CT) of the entire cranium prior to surgery, and DNA analysis for the Muenke mutation (p.Pro250Arg in FGFR3) was performed. Fourteen children had NS-UCS and the remaining 7 had MS-UCS. Table 1 summarizes patients' demographics. Twelve of the included infants had been included in 2 previous studies by this research group (Öwall et al., 2016; Öwall et al., 2019). All data were obtained in a clinical context as part of a standardized treatment regime for these children in their craniofacial center. The study does not fulfill characteristics of a medical study according to the Medical Research Act and does not need ethical permission. The protocol of this retrospective study was approved by the Hospital District of Helsinki and Uusimaa (HUS/221/2017, §47). The study was performed in accordance with the World Medical Association Declaration of Helsinki.

\section{Data Analysis}

Data comprised presurgical CT scans from which triangulated surface meshes (see Figure 1A) representing the soft tissue facial surfaces were constructed using the Marching cubes algorithm (Lorensen and Cline, 1987) with a soft tissue intensity threshold, implemented in the Landmarker software (Darvann, 2008). To analyze soft tissue FA, a computerized method based on landmark-guided surface transformation was applied, deforming a symmetric template face (an atlas) to all facial surfaces in the sample, subsequently allowing for calculations and comparisons to be performed in a meaningful way (Hutton et al., 2003; Crum et al., 2004). The method used in this study is the same method as previously used in studies by this research group (Öwall et al., 2016; Öwall et al., 2019).

The main steps of the method are further outlined here.

To create a homogenous data set of surfaces presenting with a left-sided synostosis, surfaces of patients with a right-sided synostosis $(\mathrm{N}=9)$ were mirrored prior to analysis. A fully symmetric atlas face surface was used (see Figure 1B), containing information of left-to-right correspondence for all points on the surface. The orientation of the atlas was such that the $\mathrm{x}-, \mathrm{y}-$, and z-axes in the 3D coordinate system coincided with the transverse, vertical, and sagittal directions of the face, respectively. The MSP was inherently defined in the atlas as the yz-plane dividing it into left and right halves. Twenty-two point landmarks ( 6 in the MSP and 16 bilateral landmarks located symmetrically relative to the MSP) were also defined in the atlas (see Figure 1B), inspired by Farkas (1994). The atlas surface had been constructed previously as a mean surface (containing 27880 triangles) of 44 children with and without UCS and had been made symmetrical (Öwall et al., 2019). It should be noted that the details of the shape of the atlas does not influence the calculation of asymmetry as long as it resembles the type of surface under study (in this case human faces). Soft tissue landmarks corresponding to the 22 atlas landmarks were 
Table I. Patients' Demographics.

\begin{tabular}{lccccc}
\hline Group & Patients $(\mathrm{N})$ & Mean age at CT scan (years) & Gender: $\mathrm{F}=$ female, $\mathrm{M}=$ male & $\mathrm{F}: \mathrm{M}$ ratio & Side of synostosis: $\mathrm{R}=$ right $/ \mathrm{L}=$ left \\
\hline NS-UCS & 14 & 0.7 (range: $0.2-1.4$ ) & $10 \mathrm{~F}$ & 2.50 & $5 \mathrm{R} / 5 \mathrm{~L}$ \\
& & & $4 \mathrm{M}$ & $2 \mathrm{R} / 2 \mathrm{~L}$ \\
MS-UCS & 7 & 0.4 (range: $0.1-0.6)$ & $3 \mathrm{~F}$ & 0.75 & $0 \mathrm{R} / 3 \mathrm{~L}$ \\
& & & $4 \mathrm{M}$ & 1.63 & $5 \mathrm{R} / 2 \mathrm{~L}$ \\
Total & 21 & 0.6 (range: $0.1-1.4)$ & $8 \mathrm{M}$ & & $4 \mathrm{R} / 4 \mathrm{~L}$ \\
\end{tabular}

Abbreviations: CT, computed tomography; MS-UCS, Muenke syndrome-associated unicoronal synostosis; NS-UCS, nonsyndromic unicoronal synostosis.

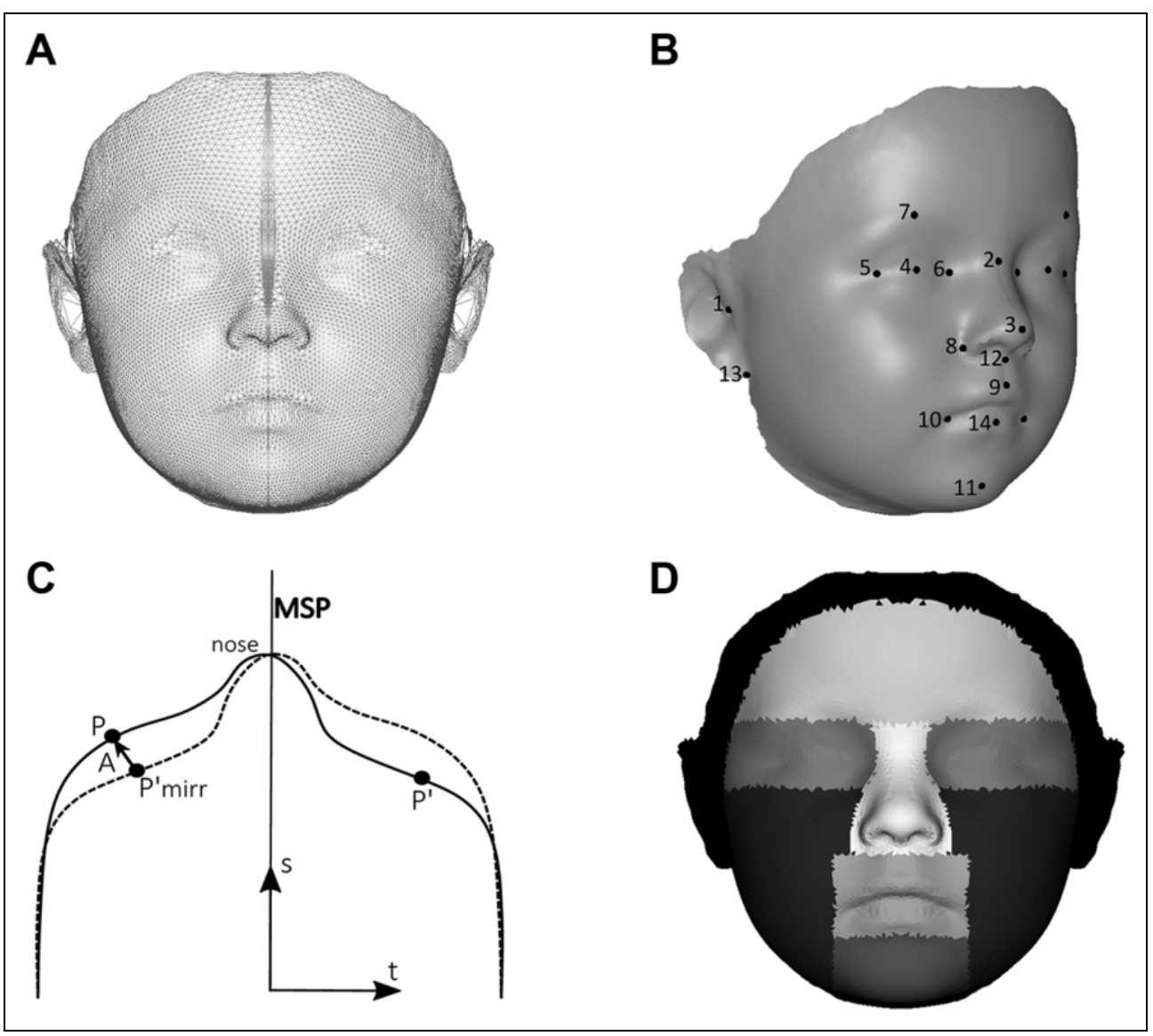

Figure I. Illustration of parts of the method. A, The atlas surface used in the present study shown as a wireframe model illustrating the density of points and triangles. B, The atlas surface with 22 soft tissue facial landmarks. Landmark names: Right tragus (I*), nasion (2), apex of nose (3), right bulbus oculi $\left(4^{*}\right)$, right lateral canthus $\left(5^{*}\right)$, right medial canthus $\left(6^{*}\right)$, right superciliare $\left(7^{*}\right)$, right alar curvature $\left(8^{*}\right)$, labiale superius $(9)$, right cheilion ( $\left.10^{*}\right)$, pogonion (I I), subnasale (I2), right otobasion inferius (I $\left.3 *\right)$, lower lip vermillion (I4), where* indicates bilateral landmarks. Landmark number I and I 3 were excluded bilaterally from orientation of the surfaces. C, The black curve illustrates a cross section of a face in the transverse plane at the level of the nose, and the dotted line illustrates the same cross section after mirroring across the midsagittal plane (MSP). Asymmetry was defined as the difference in location of an anatomical point, $\mathrm{P}$, on one side of the face and the location of the corresponding anatomical point, $\mathrm{P}$ ', on the other side of the face. Formally, facial asymmetry (amount and direction) was represented by an asymmetry vector $(A)$ between $P$ and $P$ 'mirr, which was the corresponding point on the opposite side of the face after mirroring across the MSP. The Cartesian components of the A vector represent the asymmetry in the 3 directions; transverse (t), vertical (v), and sagittal (s). D, The full face mask with its 6 subregions.

placed manually on all patient surfaces, using the Landmarker software (Darvann, 2008). Using a computer software package called Face Analyzer created in-house, based on Landmarker (Darvann, 2008) and Visualization Toolkit (Schroeder et al., 2002), the subsequent analysis was carried out.
All individual subject surfaces $(\mathrm{N}=21)$ were first oriented to the standard orientation of the atlas surface, guided by the 18 landmarks located within the facial area, excluding the landmarks located on the ears. The atlas surface was subsequently deformed by nonrigid registration, guided by all 22 landmarks 
using thin plate splines (TPS) and closest point deformation (CP), until the atlas surface was matched, virtually identically to each of the subject surfaces (Hutton et al., 2003). The resulting 21 deformed atlas surfaces contained detailed anatomical point correspondence at every point in the face and were used instead of the original surfaces in the subsequent analysis. To eliminate the difference in size between subjects, all surfaces were scaled to the same size as part of the transformation of the atlas.

Asymmetry was defined as the length (in $\mathrm{mm}$ ) and direction of an asymmetry vector (A vector), representing the difference between the same anatomical location on the left and right side of the face (see Figure 1C). The amount of asymmetry (length of the A vector) was identical on the left and right side of the face but defined to have opposite signed values on the 2 sides. The Cartesian components of the A vector corresponded to the transverse ( $\mathrm{t}$ ), vertical (v), and sagittal (s) directions of the face.

It has previously been shown (Darvann et al., 2015) that the computerized surface deformation algorithm is noisy in the peripheral regions of the face, wherefore calculations of asymmetry vectors were restricted to a region termed a full face mask (containing 16790 triangles) in the computer software. The full face mask was further divided into 6 facial subregions: forehead, eye, nose, cheek, mouth, and chin regions (see Figure 1D).

A principal component analysis (PCA) on FA was performed on all surfaces included in the study $(\mathrm{N}=21)$, using the Landmarker software (Darvann, 2008). The PCA was carried out on surfaces where the shape information had been removed from the asymmetry information, using a method inspired by Klingenberg et al. (2002). The atlas surface was deformed according to the asymmetry of each patient $(\mathrm{N}=21)$, thereby making it possible to visualize the variability of FA along particular principal component modes.

\section{Statistical Analysis}

Manual landmarking was carried out by 2 independent examiners who were both blinded to the results of each patient's genetic testing. Intraexaminer landmarking error was determined by duplicate landmarking by the same examiner (examiner 1 and 2, respectively), with a minimum of 2 to 4 weeks elapsing between the 2 sets, and interexaminer landmarking error was determined by independent landmarking by the 2 examiners. Intra- and interexaminer landmarking errors, s(i), were subsequently calculated according to Dahlberg's formula (Dahlberg, 1948).

Mean asymmetry values (including range and standard deviation) were calculated for the A vector and the 3 directions, individually and for both groups, according to the full face mask and the 6 subregions, for both examiner 1 and examiner 2 , using the computer software IDL (Interactive Data Language, Harris Geospatial Solutions Inc; Öwall et al., 2016). Systematic and random errors were evaluated using Bland-Altman plots (allowance limit set at $1 \mathrm{~mm}$ ), in IDL (Harris Geospatial Solutions Inc). Comparisons between means were carried out using Mann-Whitney $U$ (level of significance set at $5 \%$ ) and equality of variances was assessed using a nonparametric Levene's test (level of significance set at 5\%). Correlations were evaluated using Pearson rank correlation test with a level of significance of $1 \%$. Statistical analysis was performed in IBM SPSS Statistics version 24.0 (International Business Machines Corp).

\section{Results}

There was no significant difference between the mean ages in the 2 groups (see Table 1). The gender distribution in the group with NS-UCS was F:M $=2.50$, compared to 0.75 in the group with MS-UCS, indicating that the female gender was more prevalent in the group with NS-UCS, whereas the male gender was more prevalent in the group with MS-UCS. The difference was, however, not statistically significant (see Table 1). Coronal synostosis on the left side was slightly more prevalent than on the right side in the total sample and in the group with MS-UCS, whereas there was an equal left-right distribution in the group with NS-UCS. The difference between the groups was, however, not statistically significant (see Table 1).

The intraexaminer landmarking error was calculated to an average of $0.94 \mathrm{~mm}$ for examiner 1 and $0.93 \mathrm{~mm}$ for examiner 2 , and the interexaminer landmarking error was calculated to an average of $1.26 \mathrm{~mm}$. These landmarking errors were judged to be within acceptable limits. Systematic and random errors were within predefined acceptable limits.

Correlations were strong between all asymmetry calculations performed for both examiners. For examiner 1 and 2, a statistically significant difference was found according to the amount of asymmetry in the vertical direction of the chin region. For the other facial regions, there was no significant difference between the calculated FA for examiner 1 and 2, wherefore only the results from one examiner were presented.

The localization, direction, and amount of asymmetry for the respective groups were illustrated on a mean surface presenting a left-sided synostosis $(\mathrm{N}=21)$ color coded according to asymmetry. Such color maps were created for the A vector, showing the total amount of asymmetry, and its 3 Cartesian components, showing the asymmetry in the transverse, vertical, and sagittal directions. Figure 2 presents color maps for both groups and for the difference between the 2 groups. As illustrated, asymmetry was present throughout the facial region, and the global pattern of asymmetry typically observed in UCS was found in both groups. In the transverse direction, the nasal root was deviated to the ipsilateral side and the chin to the contralateral side. In the vertical direction, the forehead and eye region were elevated on the ipsilateral side, and the same areas were depressed on the contralateral side. In the sagittal direction, the forehead region was retruded on the ipsilateral side and protruded on the contralateral side. The cheek region was protruded on the ipsilateral side and retruded on the contralateral side. The group with MS-UCS evidently presented with a larger degree of asymmetry, especially in the transverse direction, compared to the group with NS-UCS (see Figure 2). 


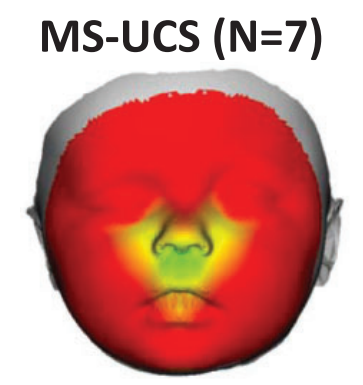

Asymmetry vector $(A)$

NS-UCS ( $N=14)$

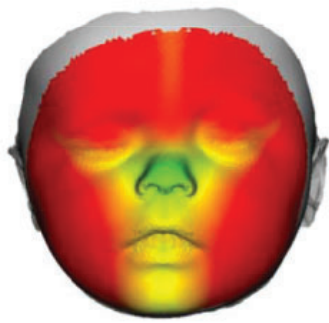

Asymmetry vector (A)

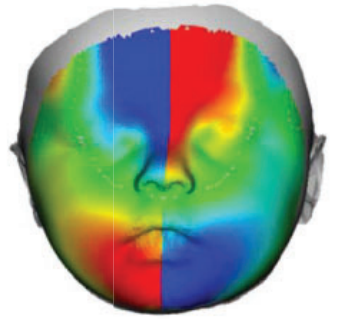

Transverse direction (t)

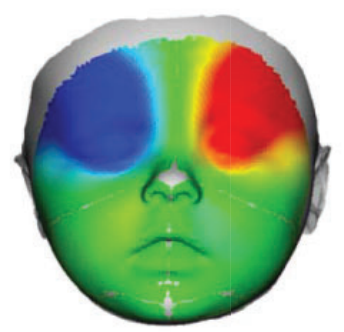

Vertical direction (v)

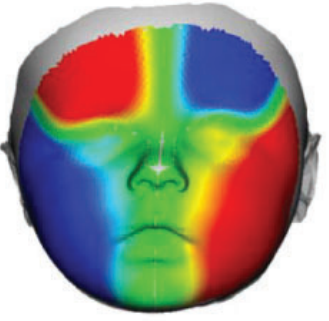

Sagittal direction (s)

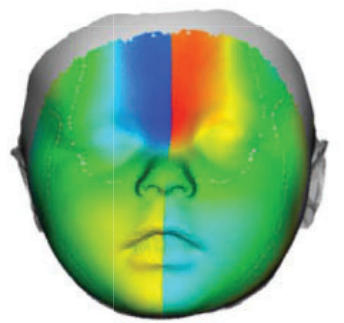

Transverse direction (t)

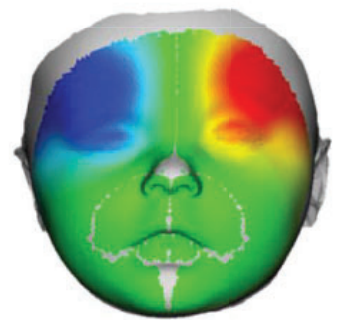

Vertical direction (v)

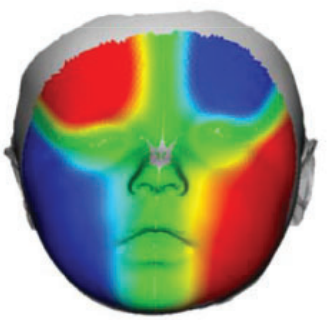

Sagittal direction (s)

\section{Difference between groups}

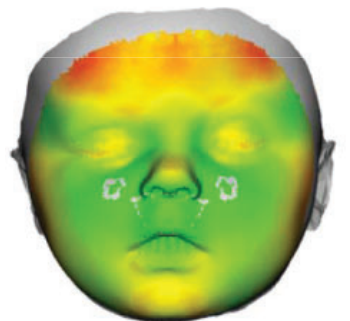

Asymmetry vector (A)

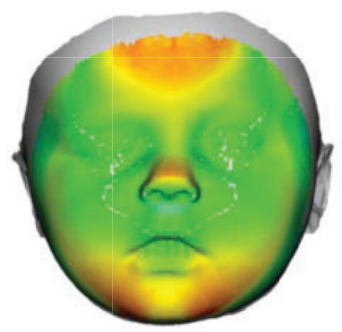

Transverse direction ( $\mathrm{t}$ )

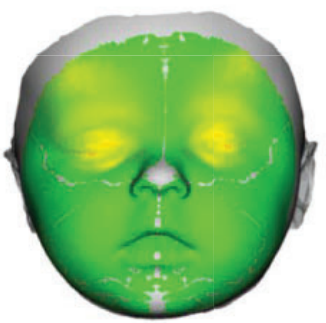

Vertical direction (v)

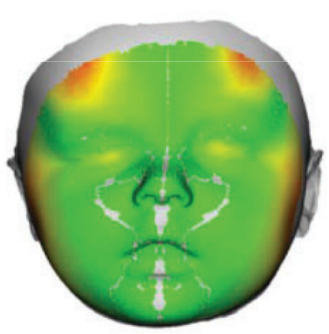

Sagittal direction (s)

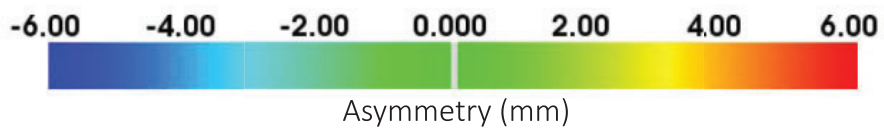

Figure 2. Facial asymmetry color maps for examiner I. The upper and middle row illustrate mean facial asymmetry for the group with Muenke syndrome-associated unicoronal synostosis (MS-UCS; $N=7$ ) and the group with nonsyndromic unicoronal synostosis (NS-UCS; $N=14$ ), respectively. The color maps for the asymmetry vector $(A)$ illustrate the distribution of the total amount of asymmetry across the facial area ranging from 0 to $6 \mathrm{~mm}$, as indicated by the color bar. In the transverse, vertical and sagittal directions the color maps illustrate the distribution, amount and direction of asymmetry as a signed value ranging from -6 to $6 \mathrm{~mm}$, as indicated by the color bar. In the transverse direction, the red color indicates an area placed further away from the midsagittal plane (MSP) compared to the corresponding area on the other side of the MSP that is placed closer to the MSP and thus colored blue. In the vertical direction, the red color indicates a superiorly displaced area compared to the corresponding area on the other side of the MSP, that is placed more inferiorly and thus colored blue. In the sagittal direction, the red color indicates an anteriorly displaced area compared to the corresponding area on the other side of the MSP that is placed more posteriorly and thus colored blue. The lower row illustrates the difference in facial asymmetry between the 2 groups (mean asymmetry in the group with MS-UCS minus mean asymmetry in the group with NS-UCS), where the asymmetry vector illustrates the total difference between the 2 groups, and the transverse, vertical, and sagittal directions illustrate the difference in the 3 directions. 
Table 2. Mean Facial Asymmetry $(\mathrm{mm})$ Corresponding to the Full Face Mask and Its 6 Subregions, for the A Vector and the Transverse, Vertical, and Sagittal Directions for Examiner I., ${ }^{\mathrm{a}, \mathrm{b}, \mathrm{d}}$

\begin{tabular}{|c|c|c|c|c|c|c|c|c|c|c|c|c|}
\hline Region and direction & \multicolumn{4}{|c|}{ MS-UCS (N = 7) } & \multicolumn{4}{|c|}{ NS-UCS $(N=14)$} & $\begin{array}{c}\text { Mean } \\
\text { difference }\end{array}$ & $P$ value $^{\mathrm{L}}$ & $P$ value ${ }^{M I}$ & $P$ value $^{M 2}$ \\
\hline A vector & 8.82 & I.II & 19.58 & 4.15 & 6.55 & 1.00 & 15.33 & 3.54 & 2.27 & .320 & .002 & .001 \\
\hline Transverse & 3.79 & $<0.01$ & 9.74 & 2.44 & 2.07 & $<0.01$ & 5.58 & 1.49 & 1.72 & .112 & $<.001$ & .001 \\
\hline Vertical & 1.87 & $<0.01$ & 9.71 & 2.59 & 1.51 & $<0.01$ & 7.55 & 1.97 & 0.36 & .942 & .156 & .456 \\
\hline A vector & 11.06 & 2.83 & 19.59 & 4.02 & 9.03 & 2.32 & 15.33 & 3.06 & 2.03 & .193 & .006 & .007 \\
\hline Transverse & 2.49 & $<0.01$ & 5.89 & $\mathrm{I} .45$ & 1.23 & $<0.01$ & 3.57 & 0.80 & 1.26 & .193 & .006 & .021 \\
\hline Vertical & 0.75 & $<0.01$ & 4.34 & 0.62 & 0.89 & $<0.01$ & 2.96 & 0.71 & -0.14 & .260 & .823 & .156 \\
\hline Sagittal & 10.08 & 1.35 & 18.69 & 4.17 & 8.30 & 1.19 & 13.82 & 2.89 & 1.78 & .233 & .014 & .009 \\
\hline \multicolumn{13}{|l|}{ Chin region } \\
\hline A vector & 6.84 & 4.86 & 8.83 & 0.88 & 3.93 & 2.47 & 7.22 & 0.98 & 2.91 & .320 & .002 & .005 \\
\hline A vector & 8.63 & 3.19 & 17.35 & 3.07 & 6.46 & 2.49 & 13.22 & 2.55 & 2.17 & .238 & .002 & .003 \\
\hline Transverse & 1.91 & $<0.01$ & 6.07 & 1.25 & 1.77 & $<0.01$ & 4.84 & 1.15 & 0.14 & .369 & .052 & .263 \\
\hline Vertical & 4.81 & 1.83 & 8.25 & 1.66 & 3.62 & 1.10 & 6.09 & 1.12 & 1.19 & .455 & .062 & .205 \\
\hline Sagittal & 5.15 & $<0.01$ & 16.39 & 4.45 & 3.72 & $<0.01$ & 12.02 & 3.34 & $\mathrm{I} .44$ & .373 & .002 & .021 \\
\hline \multicolumn{13}{|l|}{ Mouth region } \\
\hline A vector & 4.95 & I.II & 7.95 & 1.65 & 3.69 & 2.07 & 5.59 & 0.77 & 1.26 & .651 & .030 & .009 \\
\hline Transverse & 4.02 & 0.04 & 6.46 & 1.67 & 2.69 & 1.59 & 3.20 & 0.36 & 1.33 & .609 & .007 & .004 \\
\hline Vertical $^{\mathrm{e}}$ & 0.81 & $<0.01$ & 1.74 & 0.56 & 0.26 & $<0.01$ & 0.96 & 0.26 & 0.55 & .741 & .044 & .332 \\
\hline Sagittal & 1.99 & $<0.01$ & 5.14 & 1.45 & 1.99 & $<0.01$ & 4.71 & 1.29 & 0.00 & .369 & .941 & .823 \\
\hline \multicolumn{13}{|l|}{ Forehead region } \\
\hline A vector & 11.44 & 7.08 & 18.67 & 3.33 & 8.23 & 4.71 & 13.84 & 2.99 & 3.21 & .297 & .004 & .007 \\
\hline Transverse & 5.56 & $<0.01$ & 9.74 & 3.12 & 3.29 & $<0.01$ & 5.54 & 1.97 & 2.27 & .334 & .004 & .006 \\
\hline
\end{tabular}

Abbreviations: MS-UCS, Muenke syndrome-associated unicoronal synostosis; NS-UCS, nonsyndromic unicoronal synostosis; SD, standard deviation.

${ }^{a}$ Mean difference is the difference in mean asymmetry between the group with MS-UCS and the group with NS-UCS.

${ }^{\mathrm{b}} P$ value ${ }^{\mathrm{L}}$ indicates significant variances between groups assessed using nonparametric Levene test.

${ }^{C} P$ value ${ }^{M I}$ indicates significant difference between means assessed using Mann-Whitney $U$ for examiner $I$.

${ }^{\mathrm{d}} P$ value ${ }^{\mathrm{M} 2}$ indicates significant difference between means assessed using Mann-Whitney $U$ for examiner 2.

${ }^{\mathrm{e}}$ Two examiners were not in agreement.

When comparing the differences in amount of FA between the group with MS-UCS and the group with NS-UCS, there were a few discrepancies between the results from examiner 1 and 2 , as presented in Table 2. Thus, results were inconclusive when the 2 examiners were not in agreement. Significant differences between the group with MS-UCS and the group with NS-UCS confirmed by both examiners are mentioned here.

A statistically significant difference between the group with MS-UCS and the group with NS-UCS was found according to the total amount of asymmetry (A vector) in all regions studied (see Table 2). For the full face mask, a significant difference of $2.3 \mathrm{~mm}$ was calculated for the A vector. The difference was also statistically significant in the transverse and sagittal directions for the full face mask, where the transverse direction presented with the largest difference of $1.7 \mathrm{~mm}$ and the sagittal direction presented with a difference of $1 \mathrm{~mm}$.

Region-wise, the largest significant difference was seen in the forehead region $(3.2 \mathrm{~mm})$ followed by the chin $(2.9 \mathrm{~mm})$, eye $(2.2 \mathrm{~mm})$, cheek $(2.0 \mathrm{~mm})$, nose $(1.7 \mathrm{~mm})$, and mouth regions $(1.3 \mathrm{~mm})$. The transverse direction presented with the largest significant difference in the chin $(3.9 \mathrm{~mm})$, forehead $(2.3 \mathrm{~mm})$, nose $(1.7 \mathrm{~mm})$, and mouth regions $(1.3 \mathrm{~mm})$. In the eye and cheek regions, the sagittal direction presented with the largest significant difference 1.4 and $1.8 \mathrm{~mm}$, respectively (see Table 2). The variance was larger in the group with MS-UCS 


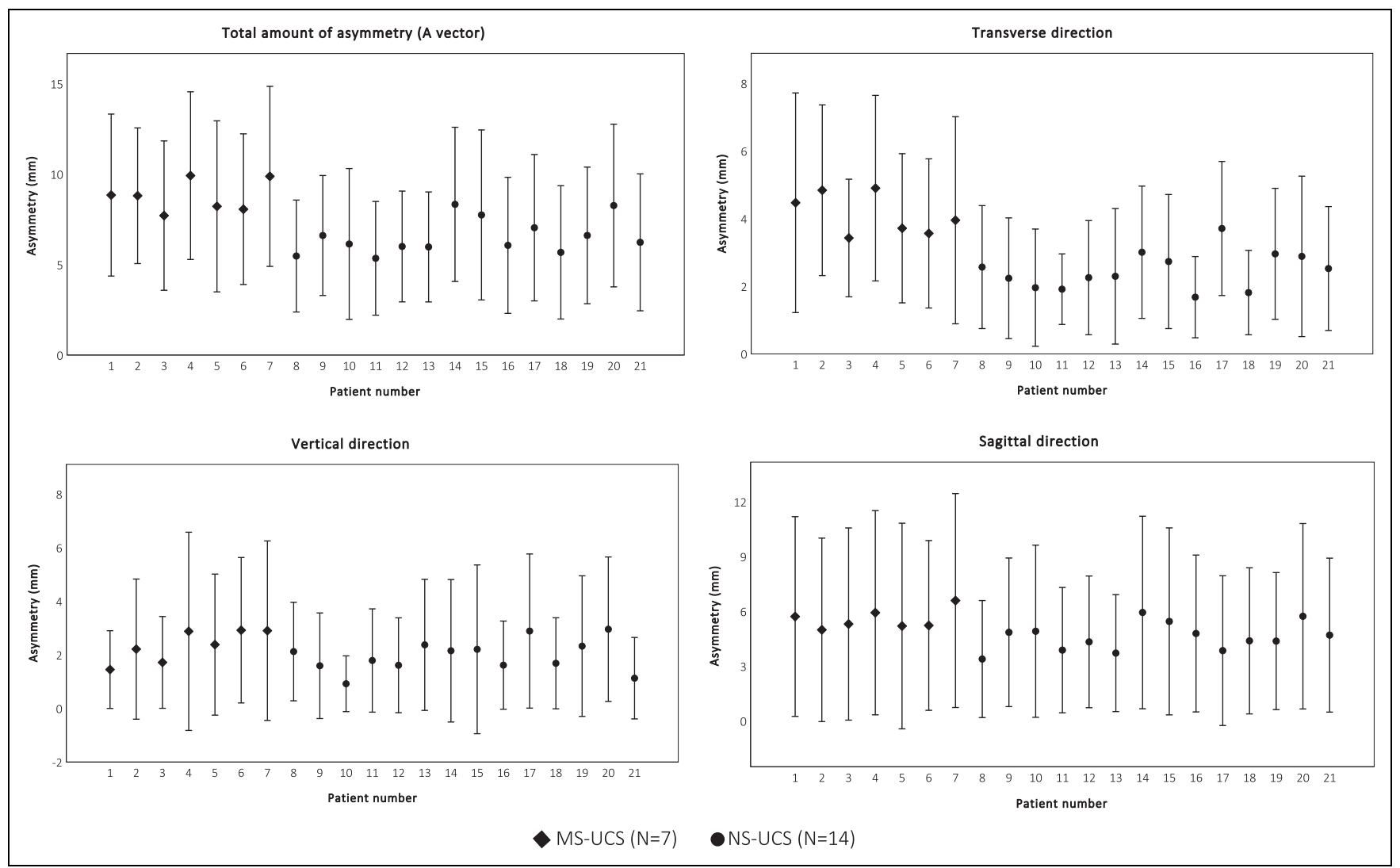

Figure 3. Individual facial asymmetry for examiner I. Graphical illustration of the individual facial asymmetry (mean \pm I standard deviation [SD]) calculated according to the full face mask, for the A vector and its 3 Cartesian components representing the transverse, vertical, and sagittal directions.

compared to the group with NS-UCS; however, the difference was not statistically significant (see Table 2).

Figure 3 presents individual mean values for the A vector according to the full face mask, for the entire sample $(\mathrm{N}=21)$, illustrating the range within and the difference between the 2 groups. There was an evident difference between the 2 groups on an individual level, especially for the A vector and the transverse direction.

There was no evidence of change in FA in regard to age at CT scanning, neither when the 2 groups were analyzed separately ( $\mathrm{N}=7$ and $\mathrm{N}=14$, respectively) nor for the sample as a whole $(\mathrm{N}=21)$.

The PCA performed on FA confirmed a statistically significant difference between the 2 groups (see Figure 4 ) when looking at the first principal component $(\mathrm{PC} 1)$ scores $(P=.001)$. A strong correlation (Pearson $r$ value: $-0.858 ; P<.001$ ) was found between $\mathrm{PC} 1$ and the calculated asymmetry corresponding to the A vector for the full face mask, indicating that PC1 captured most of the asymmetry in the sample. It was found that PC1 represented $30 \%$ of the total variance in the sample.

\section{Discussion}

Of the 7 infants with MS-UCS, 4 were males and 3 were females. Several previous studies describing gender specificity in children with MS-UCS have found a higher frequency of males than females. Muenke et al. (1997) documented that 4 of 5 individuals with MS-UCS were males; Gripp et al. (1998) 4 of 4, Lajeunie et al. (1999) 2 of 2, Casselieth et al. (2001) 6 of 6 , Doherty et al. (2007) 2 of 3, and Honnebier et al. (2008) 7 of 7. Muenke syndrome has been found to more often be associated with bicoronal synostosis (Muenke et al., 1997; Lajeunie et al., 1999; Honnebier et al., 2008; Kruszka et al., 2016), wherefore achieving larger sample sizes of children with MS-UCS is a probabilistic challenge. Despite the small sample sizes $(\mathrm{N} \leq 7)$ in all the studies mentioned above, including this present study $(\mathrm{N}=7)$, there is a uniform documentation of a male predominance in children with MS-UCS, among these 7 unrelated studies. Interestingly, bilateral coronal synostosis associated with MS is documented to occur more frequently in females (Muenke et al., 1997; Lajeunie et al., 1999; Doherty et al., 2007; Honnebier et al., 2008; Öwall et al., 2020). The explanation for these differences remains unclear.

Documentation of the side of the synostosis is not often noted in previous studies. When data are pooled from 4 studies where the side of synostosis is noted, there is a predominance of a left-sided synostosis in UCS (Gripp et al., 1998; Honnebier et al., 2008; Gonzalez-Del Angel et al., 2016; Öwall et al., 2020), which was consistent with the findings in this present study (Table 1). 


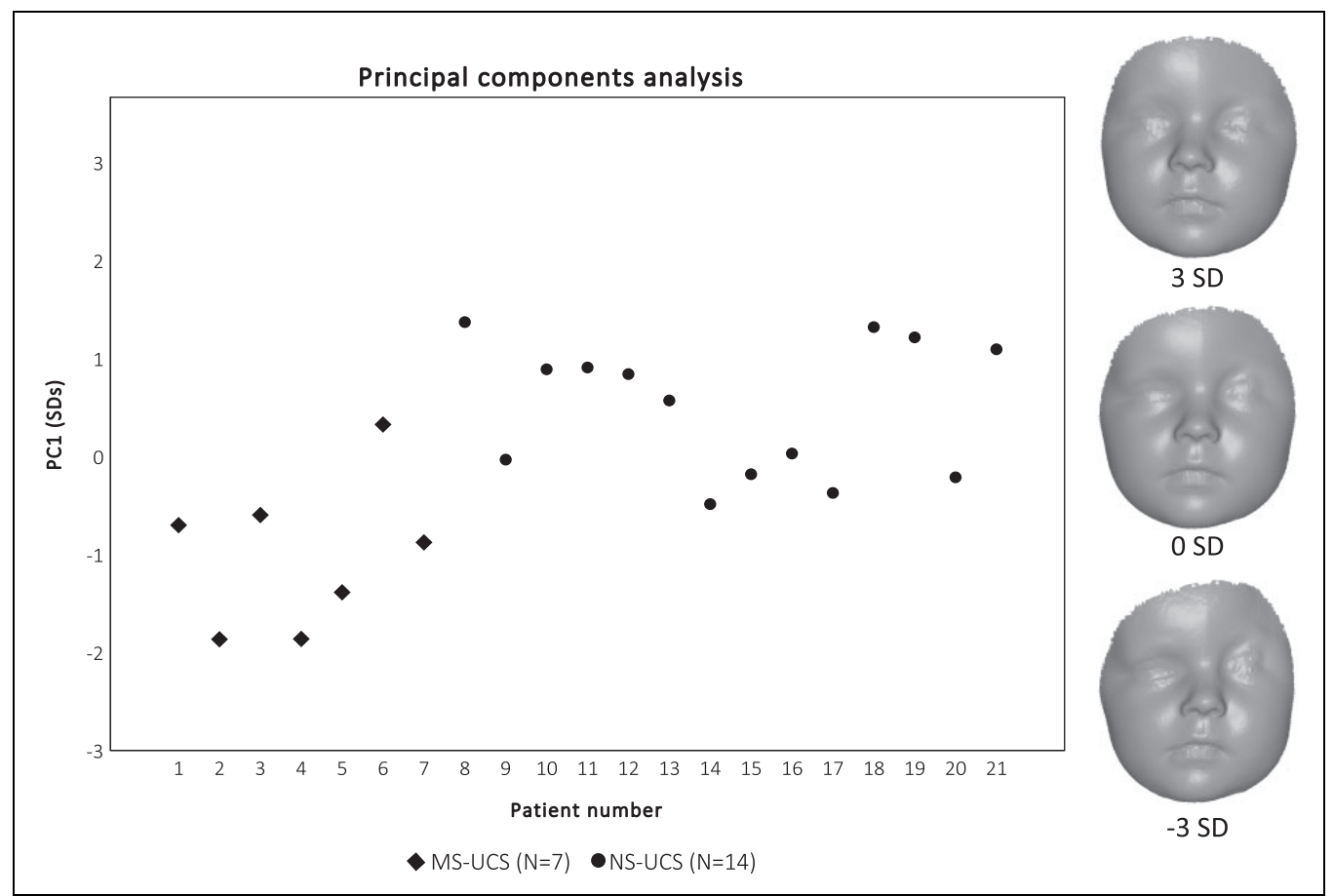

Figure 4. Principal component analysis on facial asymmetry with surface illustration for examiner I.

In this sample, there was a slight, but not significant, difference in mean age and range of age between the group with MS-UCS $(\mathrm{N}=7)$ and the group with NS-UCS $(\mathrm{N}=14)$. It could not be documented that age at CT scanning influenced the amount of FA, neither when the 2 groups were analyzed separately nor for the sample as a whole $(\mathrm{N}=21)$.

Both the group with MS-UCS and the group with NS-UCS presented with the typical facial features seen in UCS. The group with MS-UCS was, however, more severely affected throughout the facial region (see Figure 2 and Table 2). A larger degree of postoperative FA in children with MS-UCS was also found in our previous studies; however, a statistically significant difference between MS-UCS and NS-UCS could not be documented (Öwall et al., 2016; Öwall et al., 2019). Since previous studies have found poorer outcomes and increased reoperation rates in children with MS-UCS (Cassileth et al., 2001; Thomas et al., 2005; Honnebier et al., 2008; Öwall et al., 2016; Öwall et al., 2019), a difference in soft tissue FA was expected, as hypothesized. However, documentation of significant differences throughout the facial area, including the chin region was less expected and has to our knowledge not been documented previously. One of the largest differences was found in the forehead region and, interestingly, in the chin region, and the direction presenting with the largest difference throughout the facial area was the transverse direction. Underlying explanations for such differences in soft tissue FA between 2 groups with seemingly the same type of craniosynostosis can only be speculated on at this point in time. It is, however, plausible to assume that an underlying genetic cause of a condition programs the development of the cranium from an earlier fetal age, thereby resulting in a more severely affected craniofacial region in infancy.

Documentation of a more severely asymmetrical facial region in MS-UCS highlights the importance of DNA testing in all children presenting with UCS, as recommended by Warren et al. (2012), in order to supply sufficient guidance and realistic expectations to families affected by MS-UCS.

Limitations of this study include a small sample size and sources of error include landmarking error, orientation of surfaces, noise from the scanner, and inability to assure a closed mouth during acquisition of the $\mathrm{CT}$ scans in all patients, since some patients were intubated upon acquisition.

A sample size of 21 patients introduces an uncertainty in statistical analysis, wherefore this should be taken into account when reviewing our results. However, the statistical differences found between the 2 groups in this study were much strengthened by the PCA performed on FA, which clearly separated the 2 groups. Due to the rarity of UCS, a large sample size is difficult to achieve, wherefore further multicenter studies should be conducted in order to increase the sample size. Lack of reproducibility in manual placements of landmarks would introduce an error in the transformation process of the atlas in the method of this study. To eliminate the possible bias introduced from knowledge of the result of the DNA analysis, both examiners were blinded when placing the landmarks manually. Intraexaminer landmarking errors were calculated to an average of $0.94 \mathrm{~mm}$ for examiner 1 and $0.93 \mathrm{~mm}$ for examiner 2 , and the interexaminer landmarking error was calculated to an average of $1.26 \mathrm{~mm}$. These landmarking errors were considered to be within acceptable limits. Further, the transformation process did not rely solely on the manually placed landmarks for correct 
processing, but also on TPS and CP, wherefore incorrect placement of landmarks would only contribute with small errors (Hutton et al., 2003). In this study, it was found that, regardless of which examiner landmarked the surfaces, the same conclusion could be reached, namely, that a significantly larger amount of soft tissue FA in children with MS-UCS was found compared to children with NS-UCS throughout the facial region.

Since the craniofacial region is affected bilaterally in UCS, there is a lack of a normal side of the face. Therefore, we decided to orient the surfaces according to the natural head position as suggested by Tulasne and Tessier (1981), since this position is most representative of what patients experience when looking at themselves in the mirror. Orientation according to the otherwise standard orientation of a cranium, the Frankfurt-horizontal plane, would shift the MSP in the transverse plane as a result of the displaced ears in UCS, consequently increasing the calculated FA in this case. Noise from the CT scan is somehow inevitable but would not contribute substantially with errors. An open mouth during acquisition of the CT scans could result in a larger error regarding the asymmetry calculations concerning the lower areas of the face. However, this would also be an inevitable source of error, since safety of the patients should not be compromised.

The method used in this study to quantitatively assess soft tissue FA provides an objective spatially detailed evaluation of the entire facial region in 3D. Furthermore, FA could be presented both in a relatable standard of measurement (ie, millimeters) and illustrated in a color-coded manner on soft tissue surfaces. In this study, the method was applied to soft tissue facial surfaces extracted from CT scans that had been obtained as part of routine diagnostics. The method is also well suited for analysis of data obtained by 3D stereophotogrammetry, which does not subject the patient to iodizing radiation. Several similar methods for quantification of FA have been described in the recent literature (Almukhtar et al., 2016; Cho et al., 2016; Cho et al., 2018; Gabrick et al., 2020; Visse et al., 2020), which would also be well-suited methods for this patient group.

Regarding the clinical relevance of the differences in FA documented in this study, previous studies have found that FA of a similar magnitude was clinically recognizable, highlighting the relevance of the findings in this study (Farkas and Cheung, 1981; Meyer-Marcotty et al., 2011; Öwall et al., 2019). Furthermore, previously documented poorer outcomes and higher reoperation rates in children with MS-UCS also support the clinical relevance of the findings in this study (Cassileth et al., 2001; Thomas et al., 2005; Honnebier et al., 2008; Öwall et al., 2016; Öwall et al., 2019).

Comprehensive insight into the primary degree of FA in children with UCS, and differences between MS-UCS and NS-UCS, seems evident and essential in surgical planning, outcome anticipation, and patient engagement and understanding.

\section{Conclusion}

In conclusion, infants with MS-UCS presented with significantly larger FA in all regions studied throughout the facial area, compared to infants with NS-UCS. The largest significant difference was found in the forehead and chin regions, where the transverse direction presented with the largest significant difference. This study provides an objective and spatially detailed quantification of FA in 3D, in infants with MS-UCS and NS-UCS. Documentation of significantly larger FA in children with MS-UCS supports the likelihood that secondary surgeries in these children might be indicated and should probably be anticipated.

\section{Declaration of Conflicting Interests}

The author(s) declared no potential conflicts of interest with respect to the research, authorship, and/or publication of this article.

\section{Funding}

The author(s) disclosed receipt of the following financial support for the research, authorship, and/or publication of this article: This work was supported by the Lippmann foundation [grant number 500019].

\section{ORCID iDs}

Louise Öwall, MD (1) https://orcid.org/0000-0003-1857-9789

Arja Heliövaara, DDS, PhD (D) https://orcid.org/0000-0001-8064-3 484

\section{References}

Almukhtar A, Ayoub A, Khambay B, McDonald J, Ju X. State-of-theart three-dimensional analysis of soft tissue changes following Le Fort I maxillary advancement. Br J Oral Maxillofac Surg. 2016; 54(7):812-817.

Cassileth LB, Bartlett SP, Glat PM, Gripp KW, Muenke M, Zackai EH, Whitaker LA. Clinical characteristics of patients with unicoronal synostosis and mutations of fibroblast growth factor receptor 3: a preliminary report. Plast Reconstr Surg. 2001;108(7):1849-1854.

Cho MJ, Hallac RR, Ramesh J, Seaward JR, Hermann NV, Darvann TA, Lipira A, Kane AA. Quantifying normal craniofacial form and baseline craniofacial asymmetry in the pediatric population. Plast Reconstr Surg. 2018;141(3):380e-387e.

Cho MJ, Kane AA, Seaward JR, Hallac RR. Metopic 'ridge' vs. "craniosynostosis": quantifying severity with 3D curvature analysis. J Craniomaxillofac Surg. 2016;44(2):1259-1265.

Cohen MM Jr. FGFR3-associated coronal synostosis syndrome (Muenke Craniosynostosis). In: Cohen MM Jr, Maclean RE, eds. Craniosynostosis: Diagnosis, Evaluation and Management. Oxford University Press; 2000:313-315.

Crum WR, Hartkens T, Hill DL. Non-rigid image registration: theory and practice. Br J Radiol. 2004;77(2):140-153.

Dahlberg G. Statistical Methods for Medical and Biological Students. George Allen \& Unwin; 1948:122-136.

Darvann TA.Landmarker: a VTK-based tool for landmarking of polygonal surfaces. In: Takada K, Kreiborg S, eds. In Silico Dentistry: The Evolution of Computional Oral Health Science. Medigit; 2008: pp.160-162.

Darvann TA, Öwall L, Larsen P, Hove HD, Kreiborg S. Using CT to validate measurement of facial asymmetry in surface scans of infants with unilateral coronal synostosis. 2015. European Craniofacial Congress; June 24-27, 2015; Gothenburg, Sweden. 
Derderian C, Seaward J. Syndromic craniosynostosis. Semin Plast Surg. 2012;26(2):64-75.

Doherty ES, Lacbawan F, Hadley DW, Brewer C, Zalewski C, Kim HJ, Solomon B, Rosenbaum K, Domingo DL, Hart TC, et al. Muenke syndrome (FGFR3-related craniosynostosis): expansion of the phenotype and review of the literature. Am J Med Genet A. 2007;143A(24):3204-3215.

Farkas LG. Examination. In: Farkas LG, ed. Anthropometry of the Head and Face. Raven Press; 1994: pp.3-57.

Farkas LG, Cheung G. Facial asymmetry in healthy North American Caucasians. An anthropometrical study. Angle Orthod. 1981; 51(2):70-77.

Gabrick KS, Wu RT, Singh A, Bartlett SP, Taylor JA, Persing JA, Alperovich M. Assessing facial asymmetry in postoperative patients with unilateral coronal craniosynostosis. J Craniofac Surg. 2020;31(4):1000-1005.

Gonzalez-Del Angel A, Estandia-Ortega B, Alcantara-Ortigoza MA, Martinez-Cruz V, Gutierrez-Tinajero DJ, Rasmussen A, GomezGonzalez CS. Expansion of the variable expression of Muenke syndrome: hydrocephalus without craniosynostosis. Am J Med Genet A. 2016;170(12):3189-3196.

Gripp KW, McDonald-McGinn DM, Gaudenz K, Whitaker LA, Bartlett SP, Glat PM, Cassileth LB, Mayro R, Zackai EH, Muenke $\mathrm{M}$. Identification of a genetic cause for isolated unilateral coronal synostosis: a unique mutation in the fibroblast growth factor receptor 3. J Pediatr. 1998;132(4):714-716.

Honnebier MB, Cabiling DS, Hetlinger M, McDonald-McGinn DM, Zackai EH, Bartlett SP. The natural history of patients treated for FGFR3-associated (Muenke-type) craniosynostosis. Plast Reconstr Surg. 2008;121(3):919-931.

Hutton TJ, Buxton BF, Hammond P, Potts HW. Estimating average growth trajectories in shape-space using kernel smoothing. IEEE Trans Med Imaging. 2003;22(6):747-753.

Kane AA, Lo LJ, Vannier MW, Marsh JL. Mandibular dysmorphology in unicoronal synostosis and plagiocephaly without synostosis. Cleft Palate Craniofac J. 1996;33(5):418-423.

Keller MK, Hermann NV, Darvann TA, Larsen P, Hove HD, Christensen L, Schwartz M, Marsh JL, Kreiborg S. Craniofacial morphology in Muenke syndrome. J Craniofac Surg. 2007;18(2): 374-386.

Klingenberg CP, Barluenga M, Meyer A. Shape analysis of symmetric structures: quantifying variation among individuals and asymmetry. Evolution. 2002;56(10):1909-1920.

Kreiborg S, Björk A. Craniofacial asymmetry of a dry skull with plagiocephaly. Eur J Orthod. 1981;3(3):195-203.

Kreiborg S, Møller E, Björk A. Skeletal and functional craniofacial adaptations in plagiocephaly. J Craniofac Genet Dev Biol Suppl. 1985;1:199-210.

Kruszka P, Addissie YA, Yarnell CM, Hadley DW, Guillen Sacoto MJ, Platte P, Paelecke Y, Collmann H, Snow N, Schweitzer T, et al. Muenke syndrome: an international multicenter natural history study. Am J Med Genet A. 2016;170A(4):918-929.

Lajeunie E, El Ghouzzi V, Le Merrer M, Munnich A, Bonaventure J, Renier D. Sex related expressivity of the phenotype in coronal craniosynostosis caused by the recurrent P250R FGFR3 mutation. J Med Genet. 1999;36(1):9-13.
Lo LJ, Marsh JL, Pilgram TK, Vannier MW. Plagiocephaly: differential diagnosis based on endocranial morphology. Plast Reconstr Surg. 1996;97(2):282-291.

Lorensen WE, Cline HE. Marching cubes: a high resolution 3D surface construction algorithm. SIGGRAPH '87 Proceedings of the 14th Annual Conference on Computer Graphics and Interactive Techniques; New York, NY; August 1987; pp. 163-169.

Marsh JL, Gado MH, Vannier MW, Stevens WG. Osseous anatomy of unilateral coronal synostosis. Cleft Palate J. 1986;23(2):87-100.

Meyer-Marcotty P, Stellzig-Eisenhauer A, Bareis U, Hartmann J, Kochel J. Three-dimensional perception of facial asymmetry. Eur J Orthod. 2011;33(6):647-653.

Moderie C, Govshievich A, Papay F, Fearon J, Gosain A, Doumit G. Current trends in management of nonsyndromic unilateral coronal craniosynostosis: a cross-sectional survey. Plast Reconstr Surg Glob Open. 2019;7(5):e2229.

Muenke M, Gripp KW, McDonald-McGinn DM, Gaudenz K, Whitaker LA, Bartlett SP, Markowitz RI, Robin NH, Nwokoro $\mathrm{N}$, Mulvihill JJ, et al. A unique point mutation in the fibroblast growth factor receptor 3 gene (FGFR3) defines a new craniosynostosis syndrome. Am J Hum Genet. 1997;60(3):555-564.

Öwall L, Darvann TA, Hove HB, Bøgeskov L, Kreiborg S, Hermann NV. Spatially detailed 3D quantification of improved facial symmetry after surgery in children with unicoronal synostosis. Cleft Palate Craniofac J. 2019;56(7):918-928.

Öwall L, Darvann TA, Larsen P, Hove HB, Hermann NV, Bøgeskov L, Kreiborg S. Facial asymmetry in children with unicoronal synostosis who have undergone craniofacial reconstruction in infancy. Cleft Palate Craniofac J. 2016;53(4):385-393.

Öwall L, Kreiborg S, Dunø M, Hermann NV, Darvann TA, Hove HB. Phenotypic variability in muenke syndrome-observations from five Danish families. Clin Dysmorphol. 2020;29(1):1-9.

Passos-Bueno MR, Sertié AL, Jehee FS, Fanganiello R, Yeh E. Genetics of craniosynostosis: genes, syndromes, mutations and genotypephenotype correlations. In: Rice DP, ed. Craniofacial Sutures: Development, Disease and Treatment. Karger; 2008:107-143.

Richtsmeier JT, Grausz HM, Morris GR, Marsh JL, Vannier MW. Growth of the cranial base in craniosynostosis. Cleft Palate Craniofac J. 1991;28(1):55-67.

Schroeder W, Martin K, Lorensen B. The Visualization Toolkit: An Object-Oriented Approach to 3D Graphics. Kitware, Inc; 2002.

Thomas GP, Wilkie AO, Richards PG, Wall SA. FGFR3 P250R mutation increases the risk of reoperation in apparent 'nonsyndromic' coronal craniosynostosis. J Craniofac Surg. 2005;16(3):347-354.

Tulasne JF, Tessier P. Analysis and late treatment of plagiocephaly. unilateral coronal synostosis. Scand J Plast Reconstr Surg. 1981; 15(3):257-263.

Visse HS, Meyer U, Runte C, Maas H, Dirksen D. Assessment of facial and cranial symmetry in infants with deformational plagiocephaly undergoing molding helmet therapy. J Craniomaxillofac Surg. 2020;48(6):548-554.

Warren SM, Proctor MR, Bartlett SP, Blount JP, Buchman SR, Burnett W, Fearon JA, Keating R, Muraszko KM, Rogers GF, et al. Parameters of care for craniosynostosis: craniofacial and neurologic surgery perspectives. Plast Reconstr Surg. 2012;129(3): 731-737. 